\title{
miR-101 inhibits autophagy and enhances cisplatin-induced apoptosis in hepatocellular carcinoma cells
}

\author{
YONGHUA XU*, YONG AN*, YUN WANG, CHANGHE ZHANG, HAI ZHANG, \\ CHANGJUN HUANG, HAO JIANG, XUEHAO WANG and XIANGCHENG LI

\begin{abstract}
Liver Transplantation Center, First Affiliated Hospital of Nanjing Medical University, Key Laboratory of Living Donor Liver Transplantation, Ministry of Public Health, Nanjing, Jiangsu 210029, P.R. China
\end{abstract}

Received November 23, 2012; Accepted February 12, 2013

DOI: $10.3892 /$ or.2013.2338

\begin{abstract}
Hepatocellular carcinoma (HCC) ranks third in cancer-related mortality due to late diagnosis and poor treatment options. Autophagy is a lysosome-mediated protein and organelle degradation process which is characterized by the formation of double-membrane vesicles, known as autophagosomes. Increasing evidence reveals that autophagy functions as a survival mechanism in liver cancer cells against druginduced apoptosis. In this study, we found that autophagy was suppressed by miR-101 in the HCC cell line HepG2. miR-101 inhibited autophagy via targets including RAB5A, STMN1 and ATG4D. Moreover, miR-101 enhanced apoptosis induced by cisplatin in the HepG 2 cell line. The possible mechanism of this effect may be through inhibition of autophagy. Our results indicate a novel and critical role for miR-101 and autophagy in the chemoresistance of cisplatin in HCC. We propose that gene therapy targeting miR-101/autophagy should be investigated further as a potential alternative therapeutic strategy for HCC.
\end{abstract}

\section{Introduction}

Hepatocellular carcinoma (HCC) ranks third in cancer-related mortality due to late diagnosis and poor treatment options. To date, the therapies of liver cancer include surgery, chemical therapy and target therapy, but there is no complete treatment (1). An alternative therapy for liver cancer is urgently required.

Autophagy was reported for the first time by Ashford and Porter 50 years ago (2) and it has recently gained considerable attention. Autophagy is a lysosome-mediated protein and organelle degradation process that is characterized by the formation of double-membrane vesicles, referred to as

Correspondence to: Professor Xiangcheng Li, Liver Transplantation Center, First Affiliated Hospital of Nanjing Medical University, Key Laboratory of Living Donor Liver Transplantation, Ministry of Public Health, 300 Guangzhou Road, Nanjing, Jiangsu 210029, P.R. China E-mail: drxcli@njmu.edu.cn

${ }^{*}$ Contributed equally

Key words: miR-101, autophagy, hepatocellular carcinoma, chemoresistance autophagosomes $(3,4)$. Autophagy is involved in several pathophysiological processes and contributes to numerous diseases, particularly to cancer $(5,6)$. However, the function of autophagy in cancer has yet to be fully clarified, as it acts both as a tumor suppressor and as a tumor promoter (7).

Reduced autophagy is associated with a malignant phenotype and poor prognosis of HCC and activation of autophagy contributes to the growth inhibition and cell death in human liver cancer cells $(8,9)$. Increasing evidence shows that autophagy functions as a survival mechanism in liver cancer cells against drug-induced apoptosis. Autophagy inhibition enhances apoptosis induced by ginsenoside Rk1 (10), 3-bromopyruvate (11), BO-1051 (12), etoposide (13) in HCC cell lines. Targeting the autophagy pathway is a promising therapeutic strategy to improve chemotherapy efficiency. Thus, autophagy may play a dual and apparently contradictory role in HCC and the exhibited function may likely depend on the genetic composition of the cell and environmental cues the cell is exposed to.

A number of studies have recently focused on the regulation mechanism of autophagy. For example, TGF- $\beta$ signaling pathway (14) and IFN- $\gamma$ (8) activate autophagy in HCC cells. STMN1 (Stathmin 1) (15), RAB GTPase 5A (RAB5A) (16), autophagy-related protein 4D (ATG4D) and mTOR (17) have been reported to play an important role in autophagosome formation. micro-RNAs (miRNAs), which play crucial roles in HCC development and therapy, have been linked to autophagy. miR-375 inhibits autophagy by targeting autophagy-associated gene 7 (ATG7) and impairs the viability of HCC cells under hypoxic conditions both in vivo and in vitro (18). Downregulated miR-199a-5p enhanced autophagy activation by targeting ATG7 in HCC cells (19). It is reported that miR-101 inhibits basal, etoposide- and rapamycin-induced autophagy which can sensitize breast cancer cells to 4-hydroxytamoxifen (4-OHT)mediated cell death. The targets of miR-101 in this process are STMN1, RAB5A and ATG4D (15). miR-101 sensitized HCC cell lines to chemotherapeutic drug-induced apoptosis by targeting Mcl-1 (20). However, the regulation mechanism of miR-101 and its function on autophagy in HCC remains unclear.

In this study, we report that miR-101 inhibits autophagy and enhances apoptosis induced by cisplatin in HCC cells. The targets of miR-101 are STMN1, RAB5A, ATG4D and mTOR. This study revealed that miR-101 which, inhibits autophagy, might be developed as a potential novel therapy for HCC. 


\section{Materials and methods}

Cell cultures. Human HCC cell line HepG2 was purchased from Shanghai Cell Bank (Shanghai, China) and propagated in our laboratory by culturing in Dulbecco's modified Eagle's medium (DMEM) (Invitrogen, Carlsbad, CA, USA) with 10\% fetal bovine serum (FBS) (Sigma, St. Louis, MO, USA), at $37^{\circ} \mathrm{C}$ with $5 \% \mathrm{CO}_{2}$, supplemented with $1 \%$ penicillin/streptomycin. Drug treatment included cisplatin $(0.25 \mathrm{mg} / \mathrm{ml}$, Sigma $)$ and bafilomycin (400 nM, Sigma) for the indicated times.

miRNA transfection. miRNA transfection was performed using Lipofectamine 2000 (Invitrogen). Total RNA and protein were extracted at $24 \mathrm{~h}$ post-transfection and were used for quantitative real-time PCR (qRT-PCR) and western blot analysis. miR-101-mimic, inhibitor and negative control groups were designed and synthesized by GenePharma (Shanghai, China).

Reverse transcription quantitative real-time polymerase chain reaction (RT- $q P C R$ ) for miRNA and $m R N A$ quantitation. Total RNA of cells and tissues was isolated using TRIzol reagent (Invitrogen). For miRNA quantitation, cDNA was synthesized with specific miRNA reverse transcriptase primers (Applied Biosystems) using the TaqMan MicroRNA Reverse Transcription kit (Applied Biosystems, Life Technologies Corp., CA, USA). For mRNA quantitation, cDNA was synthesized using the PrimeScript RT reagent kit (Takara, Dalian, China). Quantitative RT-PCR was performed using an ABI 7500 (Applied Biosystems) with FastStart Universal SYBR Green Master (Rox) (Roche, USA) for mRNA quantitation and with TaqMan ${ }^{\circledR}$ MicroRNA Assay kit (Applied Biosystems). The relative expression of miRNA and mRNA were calculated as the inverse $\log$ of the $\triangle \Delta C T$ (21) and normalized to U6 and $\beta$-actin. Probes for miRNA qPCR were purchased from Applied Biosystems, primers for mRNA qPCR were synthesized by Invitrogen (Shanghai, China); the sequences were: STMN1 sense: 5'-TCTGTCCCAATCTTACCA-3', antisense: 5'-GAGG CATCCAAACAAAGC-3'; RAB5A sense: 5'-GCTGGTCAAG AACGATAC-3', antisense: 5'-CTTGCTTGCCTCTGAAGT-3'; ATG4D sense: 5'-GCTGCCTGACCTCGGACTGT-3', antisense: 5'-TCTGCCCAAGCTCCACCAG-3'; mTOR sense: 5'-CGCTGTCATCCCTTTATCG-3', antisense: 5'-ATGCTCA AACACCTCCACC-3'; $\beta$-actin sense: TCACCCACACTGTG CCCATCTACGA, antisense: CAGCGGAACCGCTCATT GCCAATGG.

Cell apoptosis analysis. Cell apoptosis was assessed by flow cytometry (Becton-Dickinson, San Jose, CA, USA). For cell apoptosis, cells were treated with cisplatin at a final concentration of $10 \mu \mathrm{M}$ for $48 \mathrm{~h}$. Then, cells were collected, washed, suspended in $100 \mu \mathrm{l} 1 \mathrm{X}$ binding buffer, stained with $5 \mu \mathrm{l}$ fluorescein isothiocyanate (FITC)-Annexin V and $1 \mu \mathrm{l}$ PI at room temperature for $15 \mathrm{~min}$ in the dark. The stained cells were immediately analyzed by flow cytometry.

Luciferase reporter assay. Luciferase reporter constructs were made by ligating 60-bp-long synthetic oligonucleotides (Invitrogen, Shanghai, China) containing putative miRNA binding sites from the 3'-UTR or their mutant versions of STMN1, RAB5A, ATG4D and mTOR in XbaI-FseI sites of the pGL3-control vector (Promega). Cloning was verified by sequencing. HepG2 cells were plated at $1.5 \times 10^{5}$ cells/well in 24 -well plates $24 \mathrm{~h}$ prior to transfection. Each independent luciferase reporter plasmid (200 ng) plus $80 \mathrm{ng}$ pRL-TK (Promega) was transfected in combination with $60 \mathrm{pmol}$ of miR-101-mimic, inhibitor and controls using Lipofectamine 2000 (Invitrogen). Luciferase activity was measured $48 \mathrm{~h}$ after transfection by using the Dual-Luciferase Reporter assay system (Promega). Firefly luciferase activity was normalized to Renilla luciferase activity for each transfected well.

Western blotting. Cells were lysed using RIPA buffer with $1 \%$ PMSF on ice. The concentration of total protein was determined using a BCA kit (Keygen, Nanjing, China). Equal amounts of protein $(30 \mu \mathrm{g})$ were resolved with $10 \%$ SDS-PAGE and transferred to polyvinylidene difluoride (PVDF) membranes (Millipore, Bedford, MA, USA) using a mini trans-blot apparatus (Bio-Rad Laboratories, Hercules, CA, USA). Membranes were probed with primary antibodies for $12 \mathrm{~h}$ at $4^{\circ} \mathrm{C}$ and then incubated with secondary antibodies for $2 \mathrm{~h}$ at room temperature. The primary antibodies used were: microtubuleassociated protein light chain 3 (LC3) and RAB5A rabbit polyclonal antibodies (Novus Biologicals, Littleton, CO, USA), STMN1 and mTOR antibodies (Cell Signaling Technology, Danvers, MA, USA). ATG4D antibody (Abgent, San Diego, CA, USA). GAPDH and tubulin (Santa Cruz Biotechnology, Santa Cruz, CA, USA) were used as an internal control. The secondary antibody was purchased from Beyotime (Santa Cruz Biotechnology). Electrochemiluminescence was performed with a ChemiImager 5500 imaging system (Alpha Innotech Co., San Leandro, CA, USA).

Transmission electron microscopy. Representative images were taken of cells transfected for $72 \mathrm{~h}$ as indicated and treated for $2 \mathrm{~h}$ with $200 \mathrm{nM}$ rapamycin prior to fixation. For ultrastructural examination, liver samples $\sim 1 \mathrm{~mm}^{3}$ were fixed with $2 \% \mathrm{OsO}_{4}$ and embedded in Araldite. Ultrathin sections were stained with uranyl acetate and lead citrate and inspected using an electron microscope (JEM-1010; Jeol, Tokyo, Japan).

Statistical analysis. All experiments were repeated in triplicate. All values were the means \pm standard deviation (SD). Statistical significance was determined with a Student's t-test using SPSS 15.0. P-values $<0.05$ were considered to indicate statistically significant differences.

\section{Results}

Verification of miR-101 transfection. The sequences of miRmimic, miR-inhibitor and controls are listed in Fig. 1A. The expression levels of miR-101 were confirmed by qRT-PCR. miR-mimic-treated cells showed a 31-fold higher miR-101 expression than mimic-control treated cells, whereas miRinhibitor cells had an $85 \%$ lower expression when compared with inhibitor-control treated cells (Fig. 1B).

miR-101 targets STMN1, RAB5A, ATG4D and mTOR. To explore the regulation mechanism of miR-101, we predicted four target genes (STMN1, RAB5A, ATG4D and mTOR) which were matched with miR-101 on the website www.targetscan.org. 
A

\begin{tabular}{|c|c|c|}
\hline & & Sequence 5'-3' \\
\hline \multirow{2}{*}{ miR-101-mimic } & sense & UACAGUACUGUGAUAACUGAA \\
\hline & antisense & CAGUUAUCACAGUACUGUAUU \\
\hline \multirow[t]{2}{*}{ mimic-control } & sense & UUCUCCGAACGUGUCACGUTT \\
\hline & antisense & ACGUGACACGUUCGGAGAATT \\
\hline miR-101-inhibitor & & UUCAGUUAUCACAGUACUGUA \\
\hline inhibitor-control & & CAGUACUUUUGUGUAGUACAA \\
\hline
\end{tabular}

B

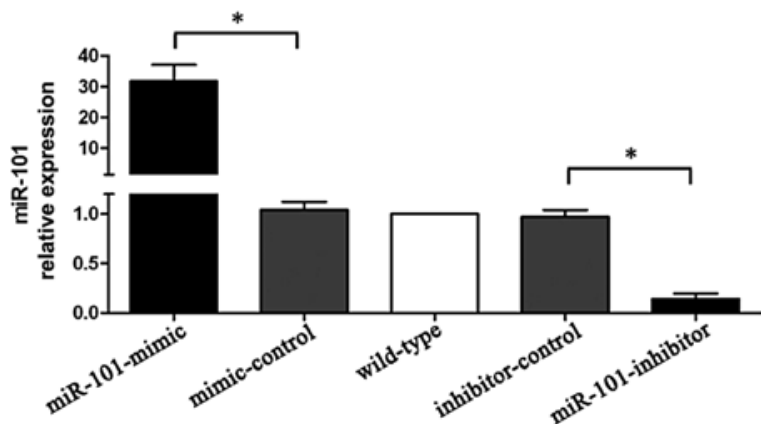

Figure 1. Verification of miR-101 transfection. (A) The sequences of miR-mimic, miR-inhibitor and controls. (B) The expression levels of miR-101 were confirmed by qRT-PCR. miR-mimic treated cells showed a 31-fold higher miR-101 expression than mimic-control treated cells, whereas miR-inhibitor cells had an $85 \%$ lower expression when compared with inhibitor-control treated cells ( $\mathrm{P}<0.05)$.

A
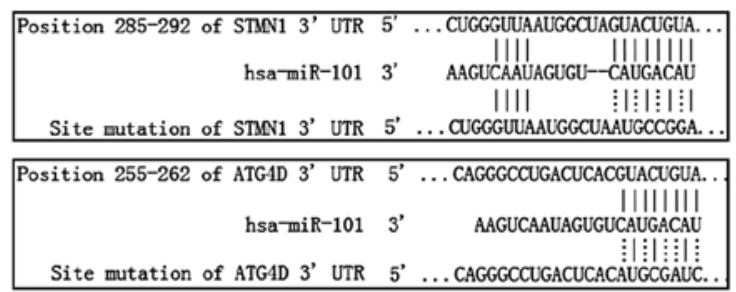

B
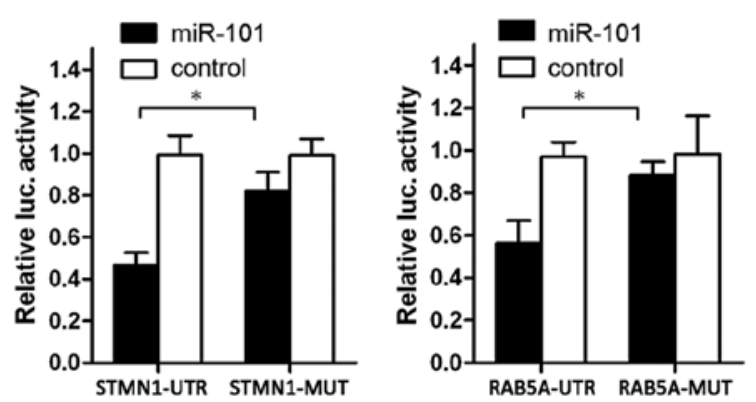

$\mathrm{C}$
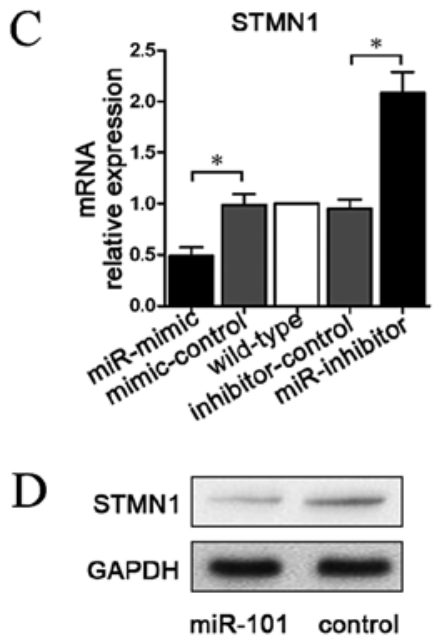
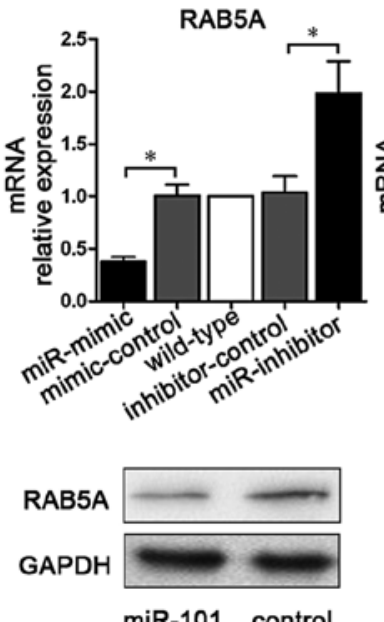
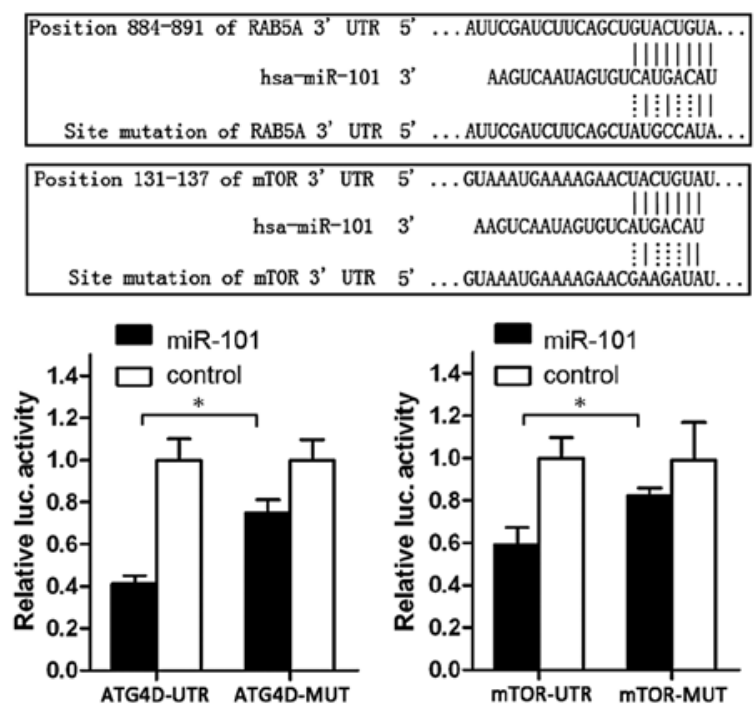

ATG4D

mTOR

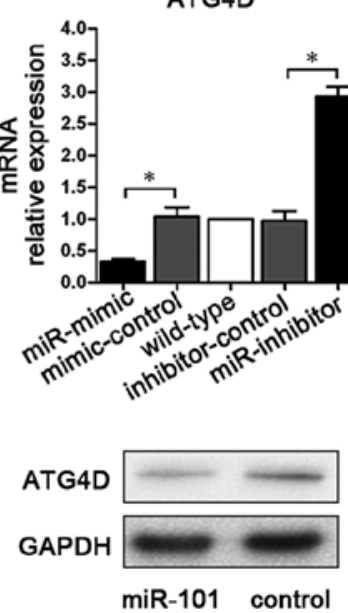

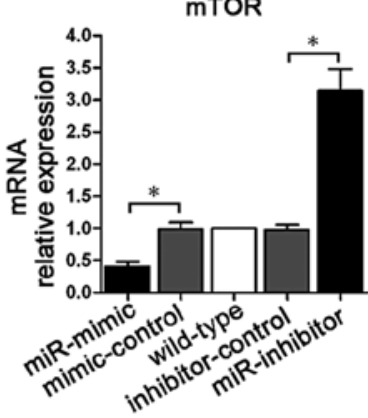

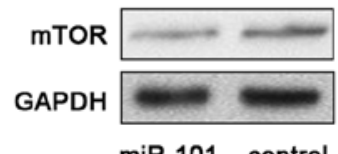

Figure 2. miR-101 targets STMN1, RAB5A, ATG4D and mTOR. (A) Four target gene (STMN1, RAB5A, ATG4D and mTOR) plasmids containing matching or mutant sequences of each gene were constructed. (B) miR-101 significantly reduced the activity of the STMN1, RAB5A, ATG4D and mTOR 3'-UTR luciferase plasmids, while plasmids containing mutant sequences were not significantly affected ("P<0.05). (C) miR-101-mimic significantly reduced STMN1, RAB5A, ATG4D and mTOR mRNA levels, whereas miR-101-inhibitor elevated the mRNA levels of these four genes ("P<0.05). (D) Western blotting showed that miR-101 downregulated the expression levels of STMN1, RAB5A, ATG4D and mTOR protein. 
A
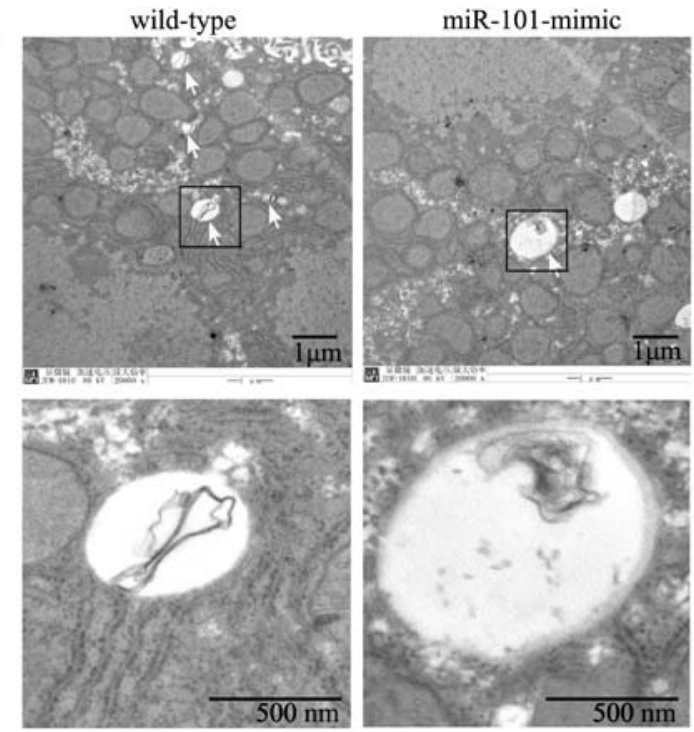

miR-101-inhibitor
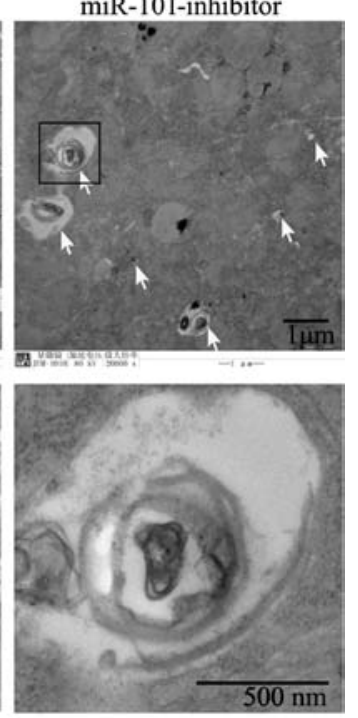

B

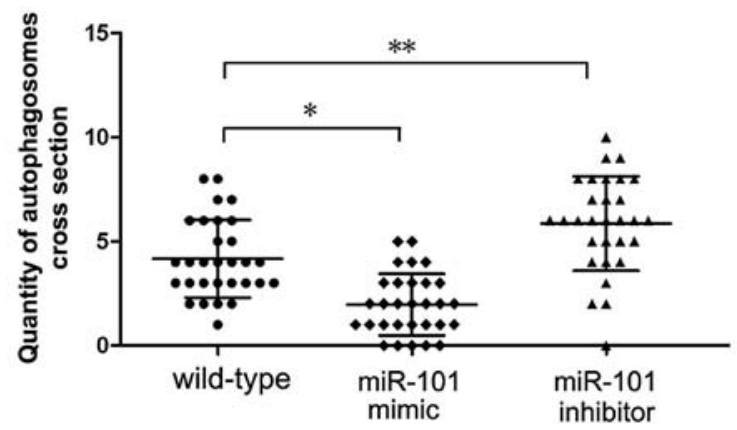

Figure 3. Transmission electron microscopy confirms suppression of autophagy by miR-101. (A) Representative images of HepG2 cells from the wild-type group, the miR-101-mimic-treated group and the miR-101-inhibitor-treated group. The white arrows indicate the double membrane-surrounded autophagosomes in the HepG2 cells. Upper panel, overview images (magnification, x20,000). Scale bars represent $1 \mu \mathrm{m}$. Lower panel, enlarged images of cytoplasmic regions of the upper panel indicated within the dashed lines. Scale bars represent $500 \mathrm{~nm}$. (B) The data were quantified by counting the number of autophagosomes per cross-sectioned cell. ${ }^{*} \mathrm{P}<0.05$ compared with wild-type ${ }^{* *} \mathrm{P}<0.01$ compared with wild-type, $\mathrm{n}=30$.

Then, plasmids containing matching or mutant sequences of each gene were constructed. These sequences are listed in Fig. 2A. To establish a direct molecular link between miR-101 and target genes, luciferase reporter assay was performed. The data showed that compared to the control group, miR-101 significantly reduced the activity of the STMN1, RAB5A, ATG4D and mTOR 3'-UTR luciferase plasmids, whereas plasmids containing mutant sequences were not significantly affected $(\mathrm{P}<0.05$; Fig. 2B). To examine the effect of miR-101 on endogenous mRNAs of STMN1, RAB5A, ATG4D and mTOR, qPCR was carried out to detect mRNA changes in miR-101-mimic transfected cells. The results showed that compared with the control group, miR-101-mimic significantly reduced STMN1, RAB5A, ATG4D and mTOR mRNA levels, whereas miR-101-inhibitor elevated the mRNA levels of these four genes $(\mathrm{P}<0.05$; Fig. $2 \mathrm{C})$. Finally, we tested the effect of miR-101 on the protein of STMN1, RAB5A, ATG4D and mTOR. As shown in Fig. 2D, miR-101 downregulated STMN1, RAB5A, ATG4D and mTOR protein. Therefore, miR-101 targets STMN1, RAB5A, ATG4D and mTOR, downregulating them both at the mRNA and at the protein level.

miR-101 suppresses autophagy. Since the formation of special double-membraned structures containing undigested cytoplasmic contents (autophagosomes) is the most important characteristic of autophagy, demonstrating these structures by electron microscopy is considered the gold standard for documenting autophagy. Hence, we examined the ultrastructural changes in HepG2 cells undergoing miR-101-mimic/inhibitor treatment. Prior to fixation, cells were transfected with miR101-mimic or miR-101-inhibitor for $72 \mathrm{~h}$. Non-transfected cells were used as a control. Quantification of autophagosomes per cellular cross-section revealed that autophagosomes were rarely detected in the non-transfected cell group. However, the number of autophagosomes was significant reduced in the miR101-mimic group (Fig. 3). LC3 is also widely used to monitor autophagy. The density of LC3-II band divided by the density of tubulin band, which represented the expression level of LC3-II. The ratio of LC3-II level/tubulin level was used as an indicator of autophagic level. In our experiment, HepG2 cells were transfected with miR-101-mimic or miR-101-inhibitor for $72 \mathrm{~h}$. Cells were treated with bafilomycin ( $400 \mathrm{nM}$, Sigma), which significantly inhibits autophagy, for $2 \mathrm{~h}$ and used as an autophagy inhibitor. Non-transfected cells were used as a control. Western blotting results showed that, compared with the non-transfected group, miR-101-mimic significantly reduced the ratio of LC3-II level/tubulin levels, suggesting the process of autophagy was inhibited. Meanwhile, miR-101-inhibitor elevated the ratio of LC3-II level/tubulin significantly (Fig. 4). Therefore, miR-101 plays the function of inhibition of autophagy. 
A

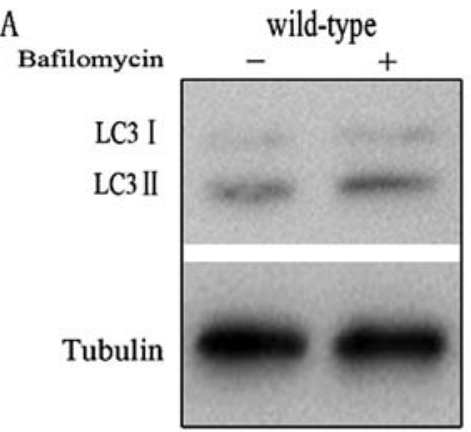

B

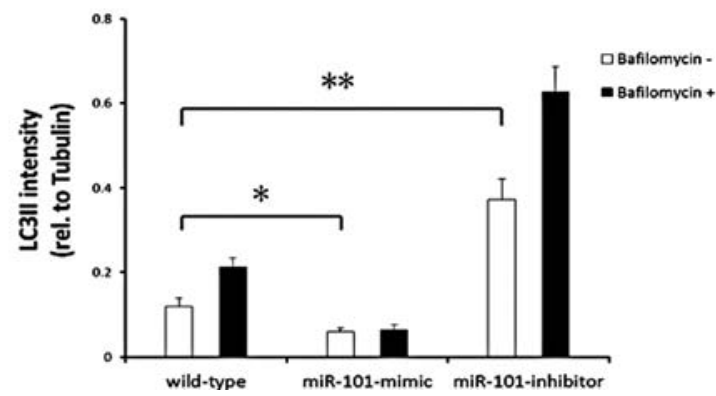

miR-101-inhibitor
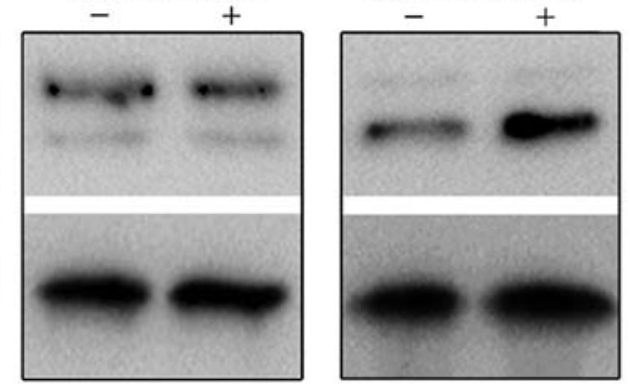

Figure 4. Western blotting confirms suppression of autophagy by miR-101. (A) Western blotting for LC3 using lysates from HepG2 cells were transfected with miR-101-mimic or miR-101-inhibitor for $72 \mathrm{~h}$. Cells were treated with $400 \mathrm{nM}$ bafilomycin for $2 \mathrm{~h}$. Non-transfected cells were used as control. The ratio of LC3-II level/tubulin level was used as an indicator of autophagic level. (B) The ratio of LC3-II/tubulin is presented in the bar charts. ${ }^{*} \mathrm{P}<0.01 \mathrm{compared}$ with untreated cells; ${ }^{* *} \mathrm{P}<0.05$ compared with untreated cells, $\mathrm{n}=3$.
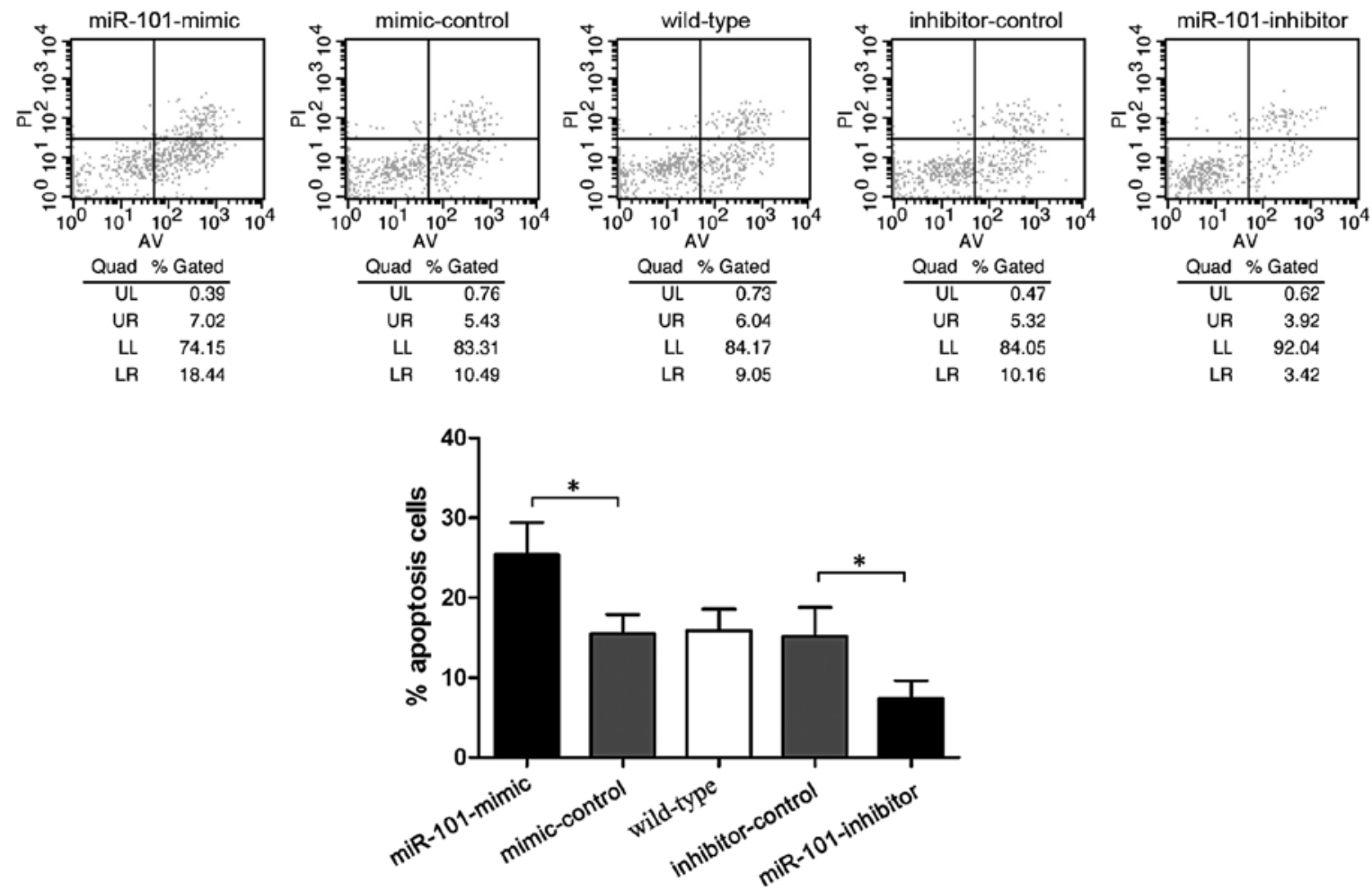

Figure 5. miR-101 enhances sensitization to cisplatin. Cisplatin-induced apoptosis was assessed by flow cytometry. Following treatment with $10 \mu \mathrm{M}$ cisplatin for $48 \mathrm{~h}$, miR-101-mimic transfected cells showed a higher apoptosis ratio (25.37 $\pm 4.05 \%)$, whereas miR-101-inhibitor exhibited a lower apoptosis ratio $\left(7.35 \pm 2.25 \%,{ }^{*} \mathrm{P}<0.05\right)$.

miR-101 enhances sensitization to cisplatin. In order to investigate the role of miR-101 in chemotherapy, cisplatin induced-apoptosis was assessed by flow cytometry. Following treatment with $10 \mu \mathrm{M}$ cisplatin for $48 \mathrm{~h}$, miR-101-mimic trans- fected cells showed a higher apoptosis ratio $(25.37 \pm 4.05 \%)$, whereas miR-101-inhibitor exhibited a lower apoptosis ratio $(7.35 \pm 2.25 \%$; $\mathrm{P}<0.05)$ (Fig. 5). This indicated that miR-101 increases cisplatin sensitivity of HepG2 cells. 


\section{Discussion}

In this study, we demonstrated that miR-101 enhances apoptosis induced by cisplatin in HCC cells. In addition, our data showed that the targets of miR-101 are STMN1, RAB5A, ATG4D and mTOR. These results suggest that miR-101 plays an important role in the cisplatin-induced apoptosis in HCC cells by inhibiting autophagy.

It is reported that miR-101 is low expressed in different types of cancer (20, 22-24). Emerging evidence suggests that miR-101 induces apoptosis, suppresses tumorigenicity and inhibits migration and invasion of gastric cancer cells (25). miR-101 sensitizes HCC cells to apoptosis and impairs the ability of cancer cells to form colonies both in vitro and in vivo (20). Moreover, myeloid cell leukemia sequence 1 (Mcl-1) was characterized as a direct target of miR-101. In addition, it was demonstrated that the TPA-induced ERK signaling pathway in HepG2 cells upregulates expression of miR-101 (23). In this study, we investigated the role of miR-101 in cisplatin-induced apoptosis of HCC cells. We found that miR-101 enhanced apoptosis induced by cisplatin in HCC cells, indicating the potential application of miR-101 in HCC therapy.

Increasing evidence shows that autophagy functions as a survival mechanism in liver cancer cells against druginduced apoptosis. A recent study showed that miRNA-101 is a potent inhibitor of autophagy in breast cancer cells. In addition, STMN1, RAB5A and ATG4D were identified as direct targets (26). In our study, miR-101 inhibited autophagy in HCC cells. Aside from STMN1, RAB5A and ATG4D, we found that mTOR was targeted directly by miR-101 as well. It is well known that suppression of mTOR leads to activation of autophagy. It seems controversial that miR-101 suppresses mTOR and inhibits autophagy. It is also well documented that several targets exist for a miRNA. Thus, the role of a miRNA is the result of changes in all of these target genes. It may be that, although we found four targets for miR-101 in autophagy, the exhibited role of miR-101 was mainly based on STMN1, RAB5A and ATG4D protein level change.

In conclusion, we showed that miR-101 plays a key role in enhancing apoptosis induced by cisplatin in HCC cells. The possible mechanism of this effect may be through inhibition of autophagy via targets including RAB5A, STMN1 and ATG4D. We propose that gene therapy targeting miR-101/autophagy should be investigated further as a potential alternative therapeutic strategy for HCC.

\section{Acknowledgements}

This study was supported by grants from the Department of Public Health of Jiangsu Province (no. RC2007056) and the National Natural Science Foundation of China (no. 81170415).

\section{References}

1. El-Serag HB and Rudolph KL: Hepatocellular carcinoma: epidemiology and molecular carcinogenesis. Gastroenterology 132: 2557-2576, 2007.

2. Ashford TP and Porter KR: Cytoplasmic components in hepatic cell lysosomes. J Cell Biol 12: 198-202, 1962.
3. Mizushima N, Levine B, Cuervo AM and Klionsky DJ: Autophagy fights disease through cellular self-digestion. Nature 451: 1069-1075, 2008.

4. Levine B and Kroemer G: Autophagy in the pathogenesis of disease. Cell 132: 27-42, 2008.

5. Mathew R, Karantza-Wadsworth V and White E: Role of autophagy in cancer. Nat Rev Cancer 7: 961-967, 2007.

6. Yang ZJ, Chee CE, Huang S and Sinicrope FA: The role of autophagy in cancer: therapeutic implications. Mol Cancer Ther 10: 1533-1541, 2011.

7. Eskelinen EL: The dual role of autophagy in cancer. Curr Opin Pharmacol 11: 294-300, 2011.

8. Li P, Du Q, Cao Z, et al: Interferon-gamma induces autophagy with growth inhibition and cell death in human hepatocellular carcinoma (HCC) cells through interferon-regulatory factor-1 (IRF-1). Cancer Lett 314: 213-222, 2012.

9. Ding ZB, Shi YH, Zhou J, et al: Association of autophagy defect with a malignant phenotype and poor prognosis of hepatocellular carcinoma. Cancer Res 68: 9167-9175, 2008.

10. Ko H, Kim YJ, Park JS, Park JH and Yang HO: Autophagy inhibition enhances apoptosis induced by ginsenoside Rk1 in hepatocellular carcinoma cells. Biosci Biotechnol Biochem 73: 2183-2189, 2009.

11. Ganapathy-Kanniappan S, Geschwind JF, Kunjithapatham R, et al: 3-Bromopyruvate induces endoplasmic reticulum stress, overcomes autophagy and causes apoptosis in human HCC cell lines. Anticancer Res 30: 923-935, 2010.

12. Chen LH, Loong CC, Su TL, et al: Autophagy inhibition enhances apoptosis triggered by $\mathrm{BO}-1051$, an $\mathrm{N}$-mustard derivative and involves the ATM signaling pathway. Biochem Pharmacol 81: 594-605, 2011.

13. Xie BS, Zhao HC, Yao SK, et al: Autophagy inhibition enhances etoposide-induced cell death in human hepatoma G2 cells. Int J Mol Med 27: 599-606, 2011.

14. Kiyono K, Suzuki HI, Matsuyama H, et al: Autophagy is activated by TGF-beta and potentiates TGF-beta-mediated growth inhibition in human hepatocellular carcinoma cells. Cancer Res 69: 8844-8852, 2009.

15. Frankel LB, Wen J, Lees M, et al: microRNA-101 is a potent inhibitor of autophagy. EMBO J 30: 4628-4641, 2011.

16. Ravikumar B,Imarisio S, Sarkar S, O'Kane CJ and Rubinsztein DC: Rab5 modulates aggregation and toxicity of mutant huntingtin through macroautophagy in cell and fly models of Huntington disease. J Cell Sci 121: 1649-1660, 2008.

17. Jung $\mathrm{CH}$, Ro SH, Cao J, Otto NM and Kim DH: mTOR regulation of autophagy. FEBS Lett 584: 1287-1295, 2010.

18. Chang Y, Yan W, He X, et al: miR-375 inhibits autophagy and reduces viability of hepatocellular carcinoma cells under hypoxic conditions. Gastroenterology 143: 177-187.e8, 2012.

19. Xu N, Zhang J, Shen C, et al: Cisplatin-induced downregulation of miR-199a-5p increases drug resistance by activating autophagy in HCC cell. Biochem Biophys Res Commun 423: 826-831, 2012.

20. Su H, Yang JR, Xu T, et al: MicroRNA-101, down-regulated in hepatocellular carcinoma, promotes apoptosis and suppresses tumorigenicity. Cancer Res 69: 1135-1142, 2009.

21. Livak KJ and Schmittgen TD: Analysis of relative gene expression data using real-time quantitative PCR and 2(-Delta Delta C(T)) Method. Methods 25: 402-408, 2001.

22. Varambally S,Cao Q, Mani RS, et al: Genomic loss of microRNA101 leads to overexpression of histone methyltransferase EZH2 in cancer. Science 322: 1695-1699, 2008.

23. Chiang CW, Huang Y, Leong KW, et al: PKCalpha mediated induction of miR-101 in human hepatoma HepG2 cells. J Biomed Sci 17: 35, 2010.

24. Buechner J, Tomte E, Haug BH, et al: Tumour-suppressor microRNAs let-7 and mir-101 target the proto-oncogene MYCN and inhibit cell proliferation in MYCN-amplified neuroblastoma. Br J Cancer 105: 296-303, 2011.

25. Wang HJ, Ruan HJ, He XJ, et al: MicroRNA-101 is downregulated in gastric cancer and involved in cell migration and invasion. Eur J Cancer 46: 2295-2303, 2010.

26. Gui $\mathrm{T}$ and Shen K: miRNA-101: A potential target for tumor therapy. Cancer Epidemiol 36: 537-540, 2012. 